\title{
Article \\ Perinatal High-Salt Diet Induces Gut Microbiota Dysbiosis, Bile Acid Homeostasis Disbalance, and NAFLD in Weanling Mice Offspring
}

\author{
Qing Guo, Yi Tang, Ying Li, Ziyuan Xu, Di Zhang, Jiangtao Liu, Xin Wang, Wei Xia * and Shunqing Xu
}

check for

updates

Citation: Guo, Q.; Tang, Y.; Li, Y.; Xu, Z.; Zhang, D.; Liu, J.; Wang, X.; Xia, W.; Xu, S. Perinatal High-Salt Diet Induces Gut Microbiota Dysbiosis, Bile Acid Homeostasis Disbalance, and NAFLD in Weanling Mice Offspring. Nutrients 2021, 13, 2135. https://doi.org/10.3390/nu13072135

Academic Editors: Albertino Bigiani and Lindsay Brown

Received: 5 March 2021

Accepted: 16 June 2021

Published: 22 June 2021

Publisher's Note: MDPI stays neutral with regard to jurisdictional claims in published maps and institutional affiliations.

Copyright: (c) 2021 by the authors. Licensee MDPI, Basel, Switzerland. This article is an open access article distributed under the terms and conditions of the Creative Commons Attribution (CC BY) license (https:// creativecommons.org/licenses/by/ $4.0 /)$.
Key Laboratory of Environment and Health (HUST), Ministry of Education \& Ministry of Environmental Protection, and State Key Laboratory of Environmental Health (Incubation), School of Public Health, Tongji Medical College, Huazhong University of Science and Technology, Wuhan 430030, China; d201881286@hust.edu.cn (Q.G.); tangyi_66237@126.com (Y.T.); D201981388@hust.edu.cn (Y.L.); M202075392@hust.edu.cn (Z.X.); M202075514@hust.edu.cn (D.Z.); D202081571@hust.edu.cn (J.L.); D201881255@hust.edu.cn (X.W.); xust@hust.edu.cn (S.X.)

* Correspondence: xiawei@hust.edu.cn; Tel.: +86-27-83693417

\begin{abstract}
A perinatal high-salt (HS) diet was reported to elevate plasma triglycerides. This study aimed to investigate the hypothesis that a perinatal HS diet predisposed offspring to non-alcoholic fatty liver disease (NAFLD), the hepatic manifestation of abnormal lipid metabolism, and the possible mechanism. Female C57BL/ 6 mice were fed a control diet $(0.5 \% \mathrm{NaCl})$ or $\mathrm{HS} \operatorname{diet}(4 \% \mathrm{NaCl})$ during pregnancy and lactation and their offspring were sacrificed at weaning. The perinatal HS diet induced greater variation in fecal microbial beta-diversity ( $\beta$-diversity) and increased bacteria abundance of Proteobacteria and Bacteroides. The gut microbiota dysbiosis promoted bile acid homeostasis disbalance, characterized by the accumulation of lithocholic acid (LCA) and deoxycholic acid (DCA) in feces. These alterations disturbed gut barrier by increasing the expression of tight junction protein (Tjp) and occludin (Ocln), and increased systemic lipopolysaccharide (LPS) levels and hepatic inflammatory cytokine secretion (TNF- $\alpha$ and IL-6) in the liver. The perinatal HS diet also inhibited hepatic expression of hepatic FXR signaling (CYP7A1 and FXR), thus triggering increased hepatic expression of pro-inflammatory cytokines (TNF- $\alpha$ and $I L-6)$ and hepatic lipid metabolism-associated genes (SREBP-1c, FAS, ACC), leading to unique characteristics of NAFLD. In conclusion, a perinatal HS diet induced NAFLD in weanling mice offspring; the possible mechanism was related to increased bacteria abundance of Proteobacteria and Bacteroides, increased levels of LCA and DCA in feces, and increased expressions of hepatic FXR signaling.
\end{abstract}

Keywords: high-salt diet; non-alcoholic fatty liver disease; hepatic inflammation; bile acid; gut microbiota

\section{Introduction}

Non-alcoholic fatty liver disease (NAFLD) has become the most common form of chronic liver disease in children and adolescents globally in recent years [1]. NAFLD in children is a major risk factor for the development of cirrhosis and hepatocellular carcinoma in adult [2,3], and it is also an early predictor of hypertension and type 2 diabetes $[4,5]$. Pregnancy is a critical period for fetal organ development [6], and maternal diet during pregnancy plays an important role in protecting against or exacerbating the offspring's risk of developing NAFLD $[7,8]$. Emerging evidence from human and animal studies has revealed that a high-salt (HS) diet in adulthood is not only a major risk factor for hypertension [9] and cardiovascular disease [10], but also exerts effects on hypertriglyceridemia [11], oxidative stress, and inflammation [12], which are well-known pathological features of the development of NAFLD $[13,14]$. In addition, a previous epidemiological study in adults showed that HS diet was associated with increased frequencies of NAFLD [15]. However, 
whether a perinatal HS diet can induce NAFLD in offspring and the related mechanism remains unknown.

Gut microbiota dysbiosis plays an essential role in the development of metabolic diseases [16], and recent studies have also implicated gut microbiota dysbiosis as an important factor in the pathogenesis of NAFLD. Fecal microbiomes of children with NAFLD showed greater variation in fecal microbial beta-diversity ( $\beta$-diversity) and higher abundance of Proteobacteria, which were demonstrated to lead to liver fibrosis [17]. Maternal diet during pregnancy could modulate the maternal microbiome and shape the gut microbiota of neonates [18]. Breast milk, which is affected by maternal diet, is also a critical factor in modulating offspring gut microbiota $[19,20]$. A previous study reported that a HS diet could increase the Firmicutes/Bacteroidetes ratio and decrease the abundance of Lactobacillus in the gut of C57BL/6J mice [21], while the changes in these gut microbiota have been reported to be associated with metabolic disease [22]. Moreover, gut microbiota can affect bile acid metabolism. Elevated lithocholic acid (LCA) and deoxycholic acid (DCA) in mice feces $[23,24]$ by gut bacteria are well-known to increase intestinal barrier permeability [25] and decrease the expression of hepatic farnesoid X receptor (FXR) [26], which are early events in the development of NAFLD [27-29]. These findings led us to hypothesize that a perinatal HS diet may interfere with offspring gut microbiota and bile acid, and then contribute to NAFLD in mice offspring.

To the best of our knowledge, this is the first study to investigate the effect of maternal HS diet during gestation and lactation on NAFLD in weanling mice offspring. We also examined gut microbiota, bile acid metabolism, and hepatic FXR signaling in the pathogenesis of NAFLD in mice offspring.

\section{Materials and Methods}

\subsection{Animal Model}

All experimental procedures were approved by the Institutional Animal Care and Use Committee of Huazhong University of Science and Technology (Wuhan, China) with a certificate of application for the Use of Animals dated 1 January 2019 (approval ID: SCXK 2015-0001). Male and female C57BL/ 6 mice were purchased from Chang Sheng biotechnology (Liaoning, China) and housed under standard environmental conditions (12 h light/dark cycle, $50-70 \%$ humidity, and $20-25{ }^{\circ} \mathrm{C}$ ). Food and water were provided ad libitum. Female C57BL/ 6 mice were divided into two groups: (1) ND group: fed a normal diet (ND) $(0.5 \% \mathrm{NaCl})$ and tap water ad libitum; (2) HS group: fed an HS diet $(4 \% \mathrm{NaCl})$ diet and tap water containing $1 \% \mathrm{NaCl}$ ad libitum. The treatment was performed from 1 week before mating until weaning of the offspring at 3 weeks of age. All diets were purchased from Beijing Huafukang Bioscience (Beijing, China). The composition and formulation of the mouse diet are detailed in Table S1. One male C57BL/ 6 mouse was paired with per two females to produce offspring. On the 14th day of gestation, pregnant mice were caged individually for delivery, and old padding was used with the cages to relieve pressure on pregnant mice. Within 5 days after delivery, the offsprings were randomly adjusted to 8 mice without hand contact with other mice. Offspring were weaned at 3 weeks of age, and body weights were recorded at 7:00 a.m. The offspring mice were fasted from 7:30 a.m. to 12:30 p.m. and stool particles were collected. After the offspring were euthanized by anesthesia with $10 \mathrm{mg} / \mathrm{mL}$ pentobarbital sodium, plasma, colon, and liver were collected and stored at $-80^{\circ} \mathrm{C}$ until biochemical analysis. Six mice per group was used for the following assays.

\subsection{Plasma and Liver Index Test}

Triglyceride (TG) and total cholesterol (TC) in the liver and plasma were measured using commercial kits (Nanjing Jiancheng Bioengineering Institute, China) that were adapted to 96-well microtiter plates. All the procedures were conducted according to the manufacturer's instructions. Absorbance was then measured at the wavelength of $450 \mathrm{~nm}$ by a microplate reader (SYNGEN, BioTek Instruments, Winooski, VT, USA). Plasma 
endotoxin (lipopolysaccharide, LPS) concentration was measured using commercial kits (Nanjing Jiancheng Bioengineering Institute, China). Absorbance was then measured at the wavelength of $580 \mathrm{~nm}$ by a microplate reader (SYNGEN, BioTek Instruments, Winooski, VT, USA).

\subsection{Liver Histological Assay}

Liver tissues were dissected on ice, fixed in $10 \%$ formalin solution, embedded in paraffin, and sectioned into 5-7 $\mu \mathrm{m}$. The slices were stained with hematoxylin and eosin (H\&E) or Masson's trichrome reagent, and then observed under a light microscope $(200 \times)$ (Olympus IX71, Tokyo, Japan). Histological analysis was quantified by the modified Pediatric NAFLD Histological Score (PNHS) [30]. Histological features were scored on a scale of $0-3$ on steatosis, $0-3$ on lobular inflammation, and 0-2 on portal inflammation. Ballooning and fibrosis were not included in the scoring, since they are characteristic features of NASH, which was not seen in this model.

\section{4. $16 \mathrm{~S}$ rRNA Gene Sequence Analysis}

The mice were placed in a sterile mouse cage lined with sterile filter paper, and stool samples were collected using sterile tweezers immediately after defecation. The filter paper was changed for each mouse sample. About $0.2 \mathrm{~g}$ ( $3 \sim 5$ grains) were collected from each mouse into sterile tubes and immediately stored at $-80^{\circ} \mathrm{C}$ until analysis. Stool DNA was extracted using the QIAamp Fast DNA Stool Mini Kit (Qiagen, CA, USA) according to manufacturer's instructions. Following DNA extraction, the V3-V4 region of the bacterial $16 \mathrm{~S}$ rRNA gene was amplified by PCR using 338F (5'-ACTCCTACGGGAGGCAGCAG-3') and 806R (5'-GGACTACHVGGGTWTCTAAT-3'), and then sequenced using the MiSeq platform (Illumina, San Diego, CA, USA). The bioinformatic-analysis readings were clustered into species-level operational taxonomic units (OTUs) at the $97 \%$ identity level. To attenuate the effect of spurious sequences, OTUs with less than $0.005 \%$ of the total number of sequences were removed [31]. After filtering, an average of 34,352 readings per sample were obtained. Calculations of within-community diversity ( $\alpha$-diversity), betweencommunity diversity ( $\beta$-diversity), relative abundance taxonomic summaries, and the different statistical analyses were preformed using QIIME. Principal coordinate analysis (PCOA) on unweighted UniFrac metrics between the HD and ND groups was tested using the ANOSIM statistical method.

\subsection{Mouse Enzyme-Linked Immunosorbent Assay (ELISA) Test}

Tumor necrosis factor (TNF)- $\alpha$ and interleukin (IL)- 6 in the liver were measured using a mouse enzyme-linked immunosorbent assay (ELISA) kit (MULTISCIENCES, Hangzhou, China). All the procedures were conducted according to the manufacturer's instructions. Absorbance was then measured at the wavelength of $450 \mathrm{~nm}$ by a microplate reader (SYNGEN, BioTek Instruments, Winooski, VT, USA).

\subsection{Bile Acid Metabolism Analyses}

Freeze-dried liver and fecal samples were weighed, $5 \mathrm{mg}$ samples were placed into $50 \mu \mathrm{L}$ of $100 \mathrm{ng} / \mathrm{mL}$ internal standard (taurochenodeoxycholic acid-d5, cholic acid-d4, gluconic acid-d4, ursodexycholic acid-d4, glycochenodeoxycholic acid-d4, glycocholic acid$\mathrm{d} 4$, lithocholic acid-d4, ursodeoxycholic acid-d4, chenodeoxycholic acid-d4, eoxycholic acid-d4, and taurocholic acid-d5) and $250 \mu \mathrm{L}$ of $0.1 \mathrm{M} \mathrm{NaOH}$. First, the mixture was reacted in an oven at $80^{\circ} \mathrm{C}$ for $1 \mathrm{~h}$. Next, the samples were placed into $700 \mu \mathrm{L}$ of $2 \%$ acetonitrile solution, and then were centrifuged at $13,000 \mathrm{rpm}$ for $5 \mathrm{~min}$ at $4{ }^{\circ} \mathrm{C}$ to collect the supernatant. After the SPE column was activated by $1 \mathrm{~mL}$ of methanol and equilibrated by $2 \mathrm{~mL}$ of $2 \%$ acetonitrile solution, $900 \mu \mathrm{L}$ of supernatant were loaded on. The column was then washed by $3 \mathrm{~mL}$ of $2 \%$ acetonitrile, $3 \mathrm{~mL}$ of n-hexane, $3 \mathrm{~mL}$ of $2 \%$ acetonitrile solution, and $4 \mathrm{~mL}$ of methanol solution, separately. Lastly, the eluents were collected, blown dry with nitrogen, and reconstituted with $150 \mu \mathrm{L}$ methanol. The bile acids from the 
liver and feces were quantitatively measured by AB Sciex API $4000^{\mathrm{TM}}$ LC/MS (Applied Biosystems, Mississauga, ON, Canada).

\section{7. qPCR RNA}

RNA was extracted from liver and colon using RNAiso Plus reagent (Takara, Kusatsu, Japan) according to the manufacturer's instructions. RNA samples were reverse-transcribed into cDNA with a HiScript ${ }^{\circledR}$ III RT SuperMix for qPCR kit (Vazyme Biotech co.ltd, Nanjing, China). The cDNA samples were amplified by qPCR with a Vazyme SYBR qPCR kit (Vazyme Biotech co.ltd, China). Each primer set was verified by analysis of their melt curves, and the assays were performed in a 7900HT Fast Real-Time PCR System (Applied Biosystems, Waltham, MA, USA). The relative gene expression was calculated by the $2-\Delta \Delta \mathrm{Ct}$ method with Gapdh as the internal control. Although studies recommended that it is better to use more than one reference gene for PCR [32], we compared multiple reference genes, and Gapdh is the most stable. The sequence of the primers used for qRT-PCR experiments is provided in Table S2. Data were analyzed with the $2-\Delta \Delta \mathrm{Ct}$ method with Gapdh gene expression used as a reference [22].

\subsection{Statistics}

Data analysis was performed using GraphPad Prism (v. 8.0). An F-test was used to test the normal distribution. Student's $t$-test was used to analyze for normally distributed data, and a Mann-Whitney $\mathrm{U}$ test was used for not normally distributed data. $p<0.05$ was considered as a significant difference and data are expressed as mean $\pm \mathrm{SEM}$.

\section{Results}

\subsection{Perinatal HS Diet Induced NAFLD in the Weanling Offspring}

Compared to the offspring with perinatal ND diet the body weights of the weanling offspring with perinatal HS diet were reduced significantly (Figure 1A), while liver weight/body weight of the offspring with perinatal HS diet displayed an increasing trend without statistical difference (Figure 1B). The perinatal HS diet did not alter plasma TC levels (Figure 1D); however, levels of plasma TG, hepatic TG, and TC were increased in the offspring with perinatal HS diet (Figure 1C,E,F). In addition, H\&E staining of liver tissue showed unique histologic features of NAFLD in the HS offspring, characterized by greater periportal leukocyte infiltration (Figure 1G). Masson staining of liver tissue showed slight fibrosis around the portal vein, while the fibrosis was not significantly different between the two groups (Figure 1H). The modified PNHS, which was used for assessing histology change in NAFLD, was significantly higher in livers of the offspring with perinatal HS diet (Figure 1I). Overall, these results showed that perinatal HS diet caused NAFLD in the weanling offspring. 

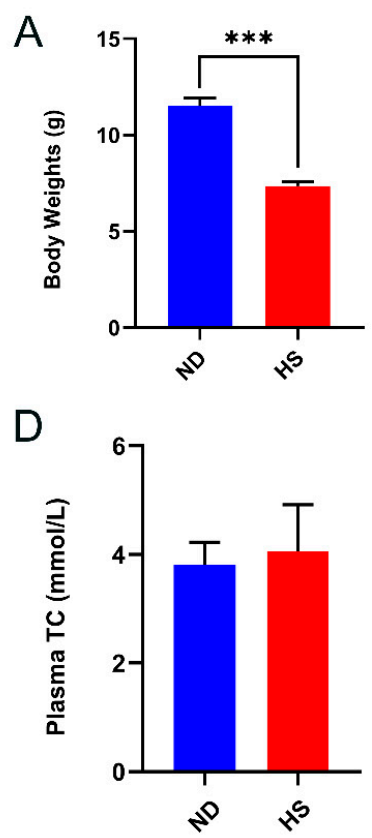

B

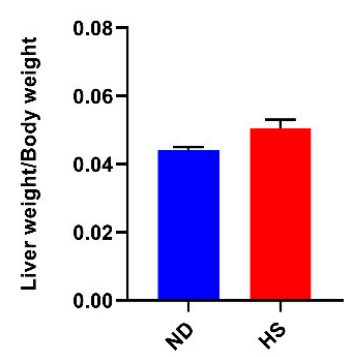

$\mathrm{E}$

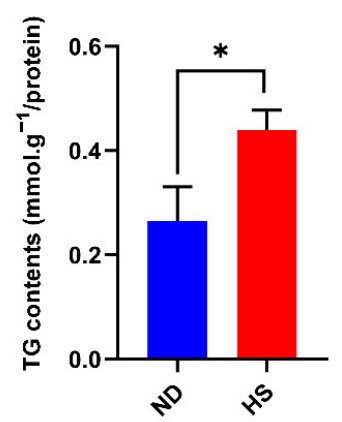

C

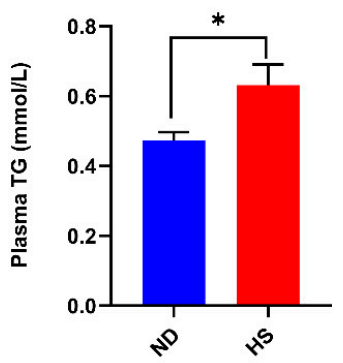

$\mathrm{F}$

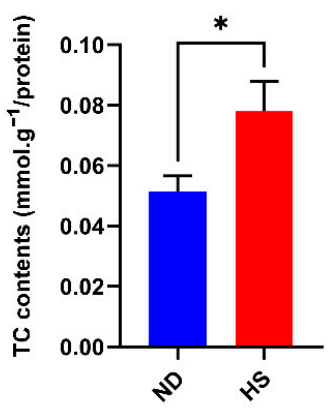

G
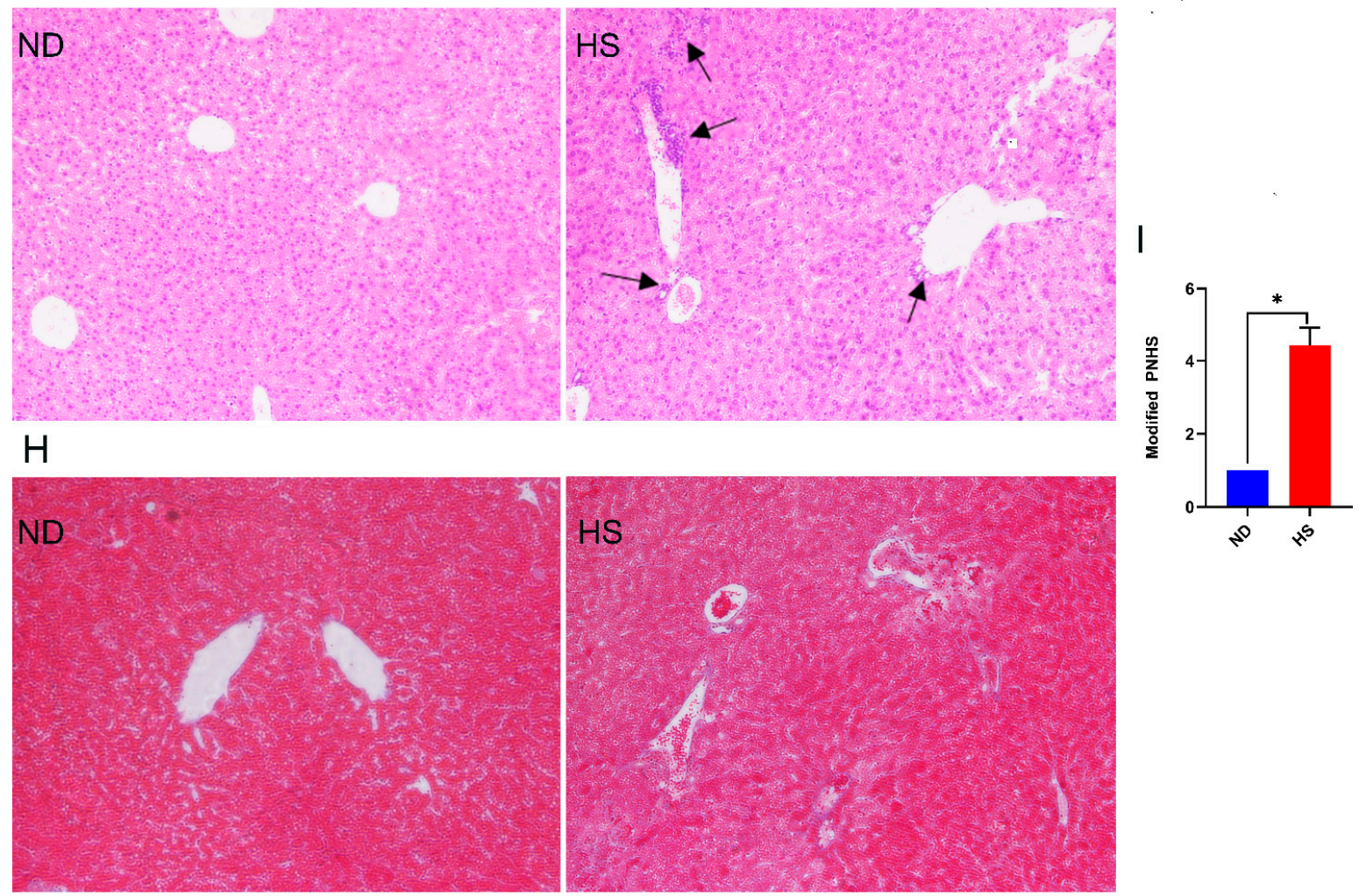

Figure 1. Effect of a perinatal high-salt (HS) diet on offspring growth, plasma triglyceride (TG), hepatic TG, hepatic total cholesterol (TC), and histological features of liver at 3 weeks of age compared to normal diet (ND). (A) Body weight. (B) Liver weight/body weight. (C) Levels of TG in plasma. (D) Levels of TC in plasma. (E) Levels of TG in liver. (F) Levels of TC in liver. (G) Representative photomicrographs of H\&E staining in the liver (200×); black arrows indicate infiltrating lymphocytes. (H) Representative photomicrographs of Masson staining in the liver (200×). (I) Modified pediatric nonalcoholic fatty liver disease (NAFLD) histological score (PNHS) for offspring of perinatal HS diet and offspring of perinatal ND diet. $n=3$ offspring of perinatal ND diet and $n=7$ for offspring of perinatal HS diet. Data are presented as mean \pm SEM. ${ }^{*} p<0.05 ; * * * 00.001$. 


\subsection{Perinatal HS Diet Altered Hepatic mRNA Expression of FXR Signaling in the Weaning Offspring}

Hepatic mRNA expression of FXR signaling, which plays an important role in the development of NAFLD, was investigated. The mRNA expressions of Fxr were significantly decreased in the livers of offspring with perinatal HS diet (Figure 2). The expression of hepatic small heterodimer partner (Shp), the downstream gene of FXR signaling, was also significantly decreased, while the expression of sterol receptor element-binding protein-1c (SREBP-1c), fatty acid synthetase (FAS), acetyl CoA carboxylase (ACC), TNF- $\alpha$, and IL-6 were increased in the livers of offspring with perinatal HS diet (Figure 2).

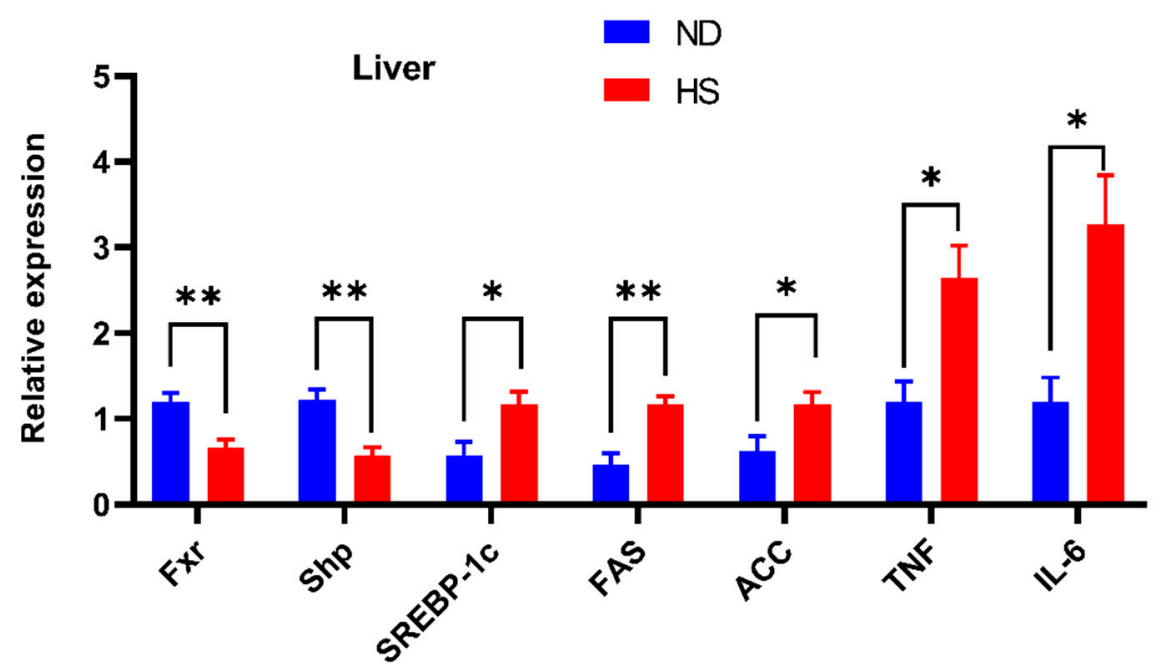

Figure 2. Effect of perinatal high-salt (HS) diet on offspring hepatic mRNA expression associated with farnesoid $X$ receptor (FXR) signaling. Relative mRNA expression of sterol receptor elementbinding protein-1c (SREBP-1c), fatty acid synthetase (FAS), acetyl CoA carboxylase (ACC), tumor necrosis factor (TNF)- $\alpha$, and interleukin (IL)-6 in the liver. The expressions were normalized to Gapdh. ${ }^{*} p<0.05 ;{ }^{* *} p<0.01$. Note: ND, normal diet.

\subsection{Perinatal HS Diet Induced Gut Microbiome Disorders in the Weanling Offspring}

Fecal microbial $\beta$-diversity showed a marked difference between offspring with perinatal HS and ND diets, based on the phylogenetic distance metric unweighted UniFrac (Figure 3A). A perinatal HS diet also led to a decreasing trend in the abundance of Verrucomicrobia and Actinobacteria phyla, and a significant increase in the abundance of Proteobacteria (Figure 3B,C). Moreover, a perinatal HS diet also led to an increasing trend in the abundance of Parabacteroides and Alloprevotella genera, and a significant increase in the abundance of Bacteroides (Figure 3D,E).

\subsection{Perinatal HS Diet Altered Bile Acid and Gut Permeability in the Weanling Offspring}

Fecal bile acid contents of LCA and DCA were significantly increased in the offspring with perinatal HS diet compared to those offspring with perinatal ND (Figure 4A). The mRNA expression levels of tight junction protein 1 (Tjp) and occludin (Ocln) in the colon were significantly decreased and the concentration of LPS was significantly increased in the offspring with perinatal HS diet (Figure 4B,C), indicating that a perinatal HS diet alters gut permeability. Consistent with the increased levels of LCA and DCA in the feces, the levels of pro-inflammatory cytokines (TNF- $\alpha$ and IL-6) were significantly increased in the livers of offspring with perinatal HS diet, while the mRNA expression of cholesterol $7 \alpha$-hydroxylase (CYP7A1), which is the ratelimiting enzyme in bile acid biosynthesis, was significantly decreased in the livers of offspring with perinatal HS diet (Figure 4D,F). Furthermore, the bile acid levels of $\alpha$-muricholic acid ( $\alpha$-MCA) and $\beta$-muricholic acid ( $\beta$-MCA) in the livers were also significantly decreased in the weanling offspring fed perinatal HS diet (Figure 4G). 


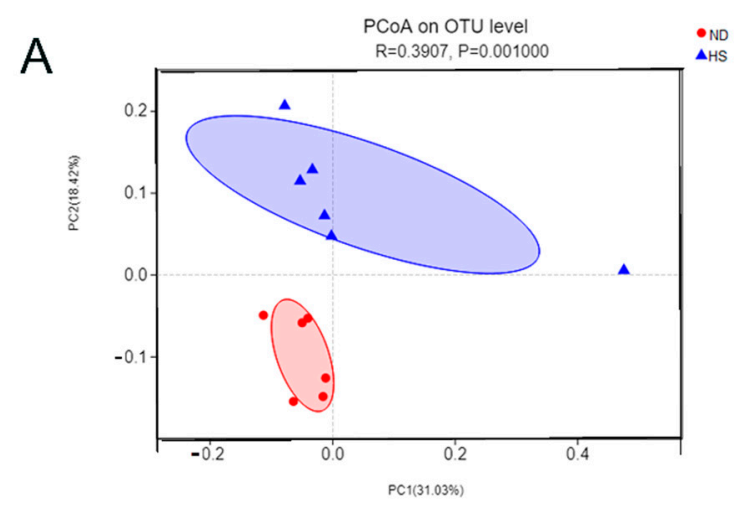

B

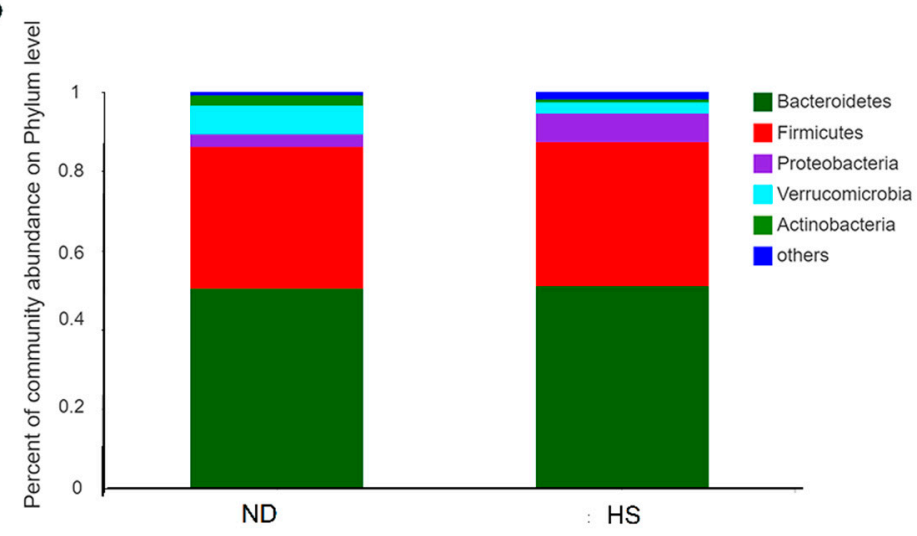

D

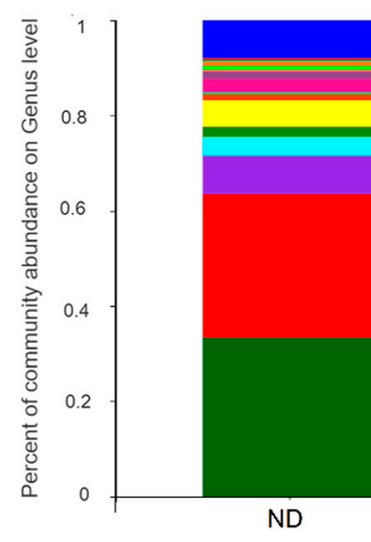

C

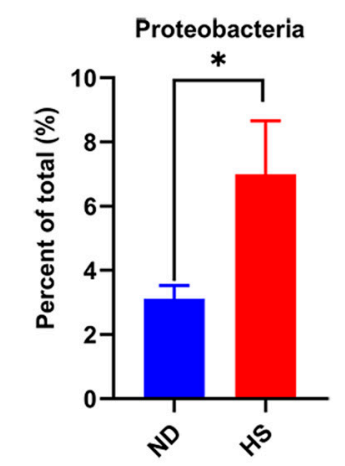

E

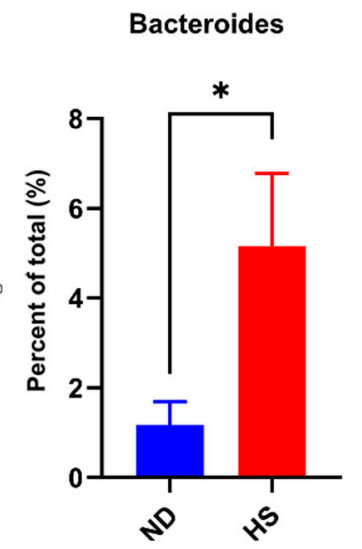

Figure 3. Effect of perinatal high-salt (HS) diet on gut microbiota of weanling offspring. (A) Principal coordinates analysis (PCoA) of unweighted Unifrac distances on the operational taxonomic unit (OTU) level. Each point represents one sample. Red dots represent normal diet (ND) samples, and blue triangles represent HS samples. Closer sample points indicate higher similarity in the species composition of the two samples. R-value represents inter-group differences and intra-group differences. A higher R-value indicates a greater difference between groups than the intragroup difference. (B) Relative abundance of bacterial phyla. (C) Relative abundances of Proteobacteria. (D) Relative abundance of bacterial genera. (E) Relative abundances of Bacteroides. ${ }^{*} p<0.05$ was considered a significant diffidence between the two groups. 
A
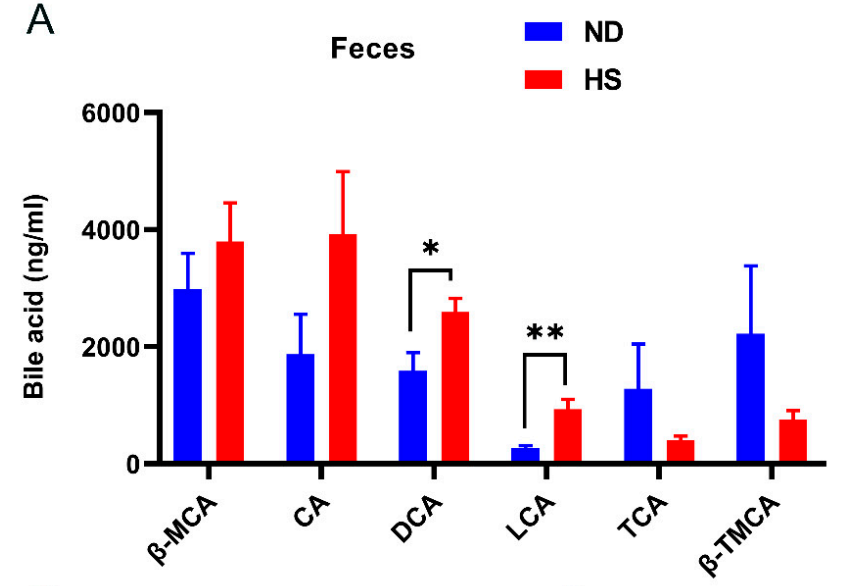

C

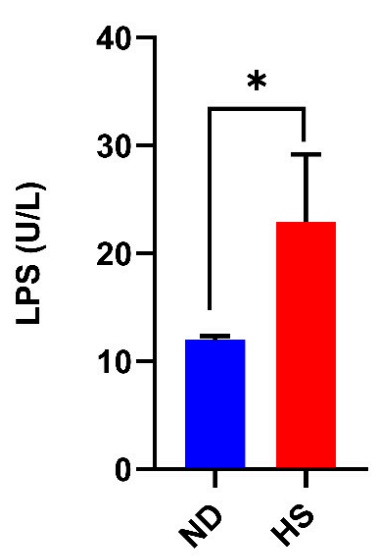

$\mathrm{F}$

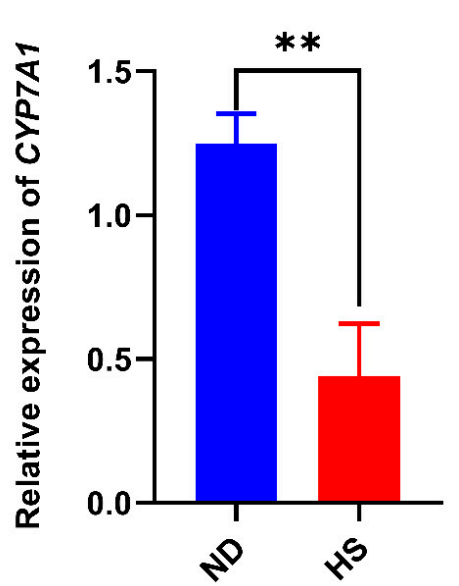

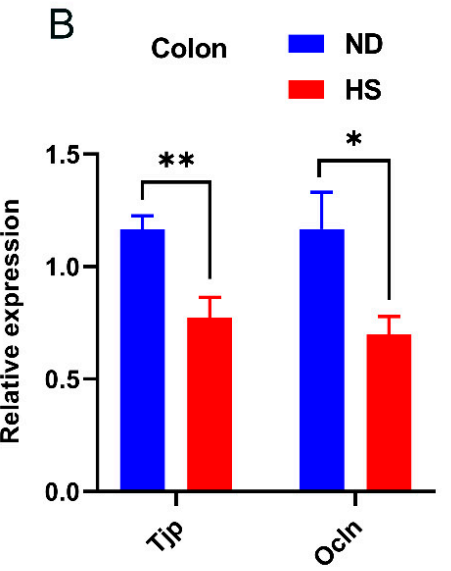

$E$

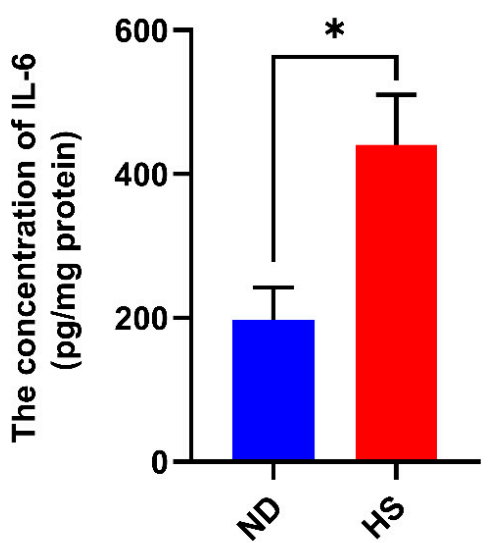

G

Figure 4. Effect of perinatal high-salt (HS) diet on offspring bile acid and gut permeability. (A) Levels of bile acid in feces. (B) Relative mRNA expression of tight junction protein (Tjp) and occludin $(\mathrm{Ocln})$ in the colon, normalized to Gapdh. (C) Plasma concentration of endotoxin (LPS). (D,E) Levels of TNF- $\alpha$ and IL-6. (F) Relative mRNA expression of CYP7A1 in the liver, normalized to Gapdh. ${ }^{*}$ indicates $p<0.05 ;{ }^{* *} p<0.01$. (G) Levels of bile acid in liver. Note: ND, normal diet; $\alpha$-MCA, $\alpha$-muricholic acid; $\beta$-MCA, $\beta$-muricholic acid; DCA, deoxycholic acid; LCA, lithocholic acid; TCA, taurocholic acid; $\beta$-TMCA, taurosulfur- $\beta$-rocholic acid.

\section{Discussion}

Perinatal diet is of critical importance for fetal development [33]. A perinatal HS diet was reported to increase plasma TG levels in mice offspring [11], which is an important indicator in the pathology of NAFLD in children [13]. In this study, we demonstrated that 
a perinatal HS diet could induce NAFLD in offspring. We also found that the perinatal HS diet increased the abundance of gut Proteobacteria and Bacteroides, and the concentration of DCA and LCA. These alterations disturbed the gut barrier by increasing expression of Tjp and Ocln [25], and increased levels of LPS and hepatic inflammatory cytokine secretion (TNF- $\alpha$ and IL-6) in the liver [34]. These changes inhibited the expression of hepatic FXR signaling (CYP7A1 and FXR), thus triggering increased hepatic expression of proinflammatory cytokines (TNF- $\alpha$ and IL-6) [35] and hepatic lipid metabolism associated genes (SREBP-1c, FAS, and ACC) [24], leading to the unique characteristics of NAFLD.

The earlier presentation of NAFLD in children characterized mainly by the presence of a predominant portal-based injury, including portal inflammation with no or mild fibrosis [2]. In this study, a perinatal HS diet led to increased hepatic periportal leukocyte infiltration. Although histologic change in the liver did not show obvious lipid deformation, the biochemical indicators of pediatric NAFLD patients appeared abnormal, including hyperlipidemia [3]. The reason for this phenomenon is the lack of accurate methods to identify liver steatosis. When the total area of steatosis is less than $20 \%$, the histologic method for identifying liver steatosis will generate a false negative [4]. Similarly, we did not find obvious TG accumulation in tissue by H\&E and Masson staining, but the levels of hepatic TG increased significantly. The modified pediatric NAFLD histological score (PNHS) [30] is a recently developed method used for assessing histology change of NAFLD to improve accuracy. Histological features were scored on a scale of $0-3$ on steatosis, $0-3$ on lobular inflammation, and 0-2 on portal inflammation. The PNHS method has also been used in other animal experiments, and it was proved that it can identify NAFLD-like lesions in weaned mice [22]. We observed PNHS was significantly higher in the livers of the offspring with perinatal HS diet. Accumulating epidemiological evidence suggests that a small body size during infancy and childhood is associated with a higher risk of developing NAFLD [36,37]. Moreover, dyslipidemia is also observed in children with NAFLD [38]. In accordance with the features of NAFLD in children, we found that perinatal HS diet led to decreased body weights and increased levels of plasma TG in the offspring. Consistent with our study, Clare et al. reported that Dawley rats fed a $4 \%$ salt diet during pregnancy and lactation induced lower body weights and higher level of plasma TG in offspring [11].

Hepatic FXR signaling plays an important role in the development of NAFLD [39]. Hepatic FXR is a bile acid receptor and can modulate lipid homeostasis via SHP. SHP is a FXR target gene and promoter-specific repressor. Downregulated expression of Fxr can repress Shp expressions, which in turn facilitates the expression of SREBP-1c [40]. SREBP-1c as a key transcription factor plays an important role in the regulation of the expression of lipogenic genes, including ACC-1 and FAS [6]. Several studies have proved that the overexpression of $S R E B P-1 c, F A S$, and $A C C$ is associated with accelerated hepatic lipogenesis in NAFLD animal models [41,42], while downregulated mRNA expression of these genes can protect development of liver steatosis $[43,44]$. Moreover, a study reported that downregulated expression of Fxr could repress Shp expression, which in turn could facilitate the expression of $S R E B P-1 c, A C C$, and $F A S$, and then result in the development of NAFLD [24]. Therefore, the upregulated expression of SREBP-1c, FAS, and ACC observed in our study suggested that perinatal HS diet induce hepatic lipogenesis and lipid accumulation. The decreased expression of FXR also increased the expression of TNF- $\alpha$ and IL-6 [35]. Hepatic lipid synthesis and inflammation are crucial mechanisms in the pathogenesis of NAFLD [45].

Substantial studies have indicated that gut microbiota dysbiosis contributes to hepatic lipid synthesis and inflammation in NAFLD $[46,47]$. Proteobacteria can increase hepatic inflammation [48] and are also a marker for gut microbiota dysbiosis and metabolic diseases in human and mice models [49]. Bacteroides are salt-tolerant bacteria [50] and are associated with liver injury in human studies [51,52]. Animal studies also showed that the abundance of Proteobacteria and Bacteroides are higher in NAFLD [53,54]. Therefore, the increased abundance of Proteobacteria and Bacteroides may suggest liver damage and increased risk for NAFLD. To determine whether changes in lipid metabolism were induced by gut 
microbiota dysbiosis, 16S rRNA analysis in our study was performed. We found perinatal HS diets increased the abundance of gut Proteobacteria and Bacteroides in weanling mice offspring. The results are consistent with previous studies that HS diets induce increases in the abundance of Proteobacteria and Bacteroides $[53,55]$.

Gut microbiota dysbiosis and its influence on bile acids were recently considered [56]. It is well known that gut microbes produce enzymes that convert primary bile acids into secondary bile acids in the intestines. Bacteroides have bile salt hydrolase activity, which can metabolize primary bile acids into secondary bile acids, such as DCA and LCA [23], which are cytotoxic. DCA and LCA can potentially increase gut permeability and increase the exposure of the liver to gut-derived toxins, which can promote hepatic inflammation and liver steatosis in animal models of NAFLD $[57,58]$. Previous studies have shown that increased abundance of Proteobacteria and Bacteroides led to elevated levels of DCA and LCA, which consequently increased levels of TNF- $\alpha$ and IL-6, and inhibited the expression of CYP7A1 in the livers of mice [59-61]. Some studies have shown that HS intake induced gut dysbiosis and disrupted gut barrier integrity, and that antibiotics could restore HSdiet-induced gut leakiness $[53,62,63]$. Furthermore, the levels of Proteobacteria, Bacteroides, DCA, LCA, TNF- $\alpha$, and IL-6 in mice could be reversed by antibiotic treatment [64]. In the classic pathway of bile acid biosynthesis, CYP7A1 determines the overall rate of bile acid production and synthesizes two primary bile acids: cholic acid and chenodeoxycholic acid (CDCA) [23]. In mice, the majority of CDCA is converted to $\alpha$-MCA and $\beta$-MCA, which are both agonistic with FXR [65]. Overexpression of Cyp7a1 can activate expression of $F x r$, whereas inhibition of the expression of $C y p 7 a 1$ can reduce bile acid synthesis and decrease the expression of $F x r[24,66]$. Owing to the decrease in the expression of Cyp7a1 in our study, we tested hepatic main bile acid. Consistent with these studies, we found that increased DCA and LCA led to gut permeability, increased the levels of hepatic TNF- $\alpha$ and IL-6, and decreased the expression of hepatic CYP7A1 and the contents of $\alpha$-MCA and $\beta$-MCA. Based on these reasons, we think that gut dysbiosis is an important link of perinatal HS diet to the progression of NAFLD in the offspring. Future studies need to be confirmed by antibiotic experiments.

\section{Conclusions}

In conclusion, this study revealed that a perinatal HS diet contributed to NAFLD in weanling offspring. Taken together, the perinatal HS diet increased the abundance of Proteobacteria and Bacteroides and elevated the fecal LCA and DCA levels, thereby destroying the gut barrier, increasing the levels of hepatic TNF and IL-6, and inhibiting the expression of CYP7A1 and Fxr, which induced hepatic inflammation and lipid accumulation, potentially contributing to the progression of NAFLD in the weanling offspring. The findings of this study expand our current knowledge of the effect of perinatal HS intake on NAFLD of weanling offspring and the role of gut microbiota on NAFLD of weanling offspring. Further studies are needed to explore whether treatment with probiotics via modulation bile acids can improve disease progress in NAFLD of weanling offspring.

Supplementary Materials: The following are available online at https://www.mdpi.com/article/10 .3390/nu13072135/s1, Table S1: The compositions and formulas of normal diet and high-salt diet, Table S2: Primer sequences.

Author Contributions: Q.G. designed the study, performed all experiments, and wrote the manuscript. Y.T. designed the study and helped carry out the experiments. Y.L., Z.X., D.Z., J.L. and X.W. helped carry out the experiments. S.X. designed the study and supervised all experiments. W.X. designed the study and supervised all experiments, writing-review and editing. All authors have read and agreed to the published version of the manuscript.

Funding: This work was supported by the National Natural Science Foundation of China (91643207, and 21437002); the Fundamental Research Funds for the Central Universities, Huazhong University of Science and Technology (2015ZDTD047, 2016YXZD043, and 2018KFYXMPT00); and the Program for HUST Academic Frontier Youth Team (2018QYTD06 and 2018QYTD12). 
Institutional Review Board Statement: All experimental procedures were approved by the Institutional Animal Care and Use Committee of Huazhong University of Science and Technology (Wuhan, China) with a certificate of application for the Use of Animals dated 1 January 2019 (approval ID: SCXK 2015-0001).

Data Availability Statement: The data presented in this study are available on request from the corresponding author.

Conflicts of Interest: The authors declare no conflict of interest.

\section{References}

1. Schwimmer, J.B.; Deutsch, R.; Kahen, T.; Lavine, J.E.; Stanley, C.; Behling, C.J.P. Prevalence of fatty liver in children and adolescents. Pediatrics 2006, 118, 1388-1393. [CrossRef] [PubMed]

2. Goyal, N.P.; Schwimmer, J.B. The Progression and Natural History of Pediatric Nonalcoholic Fatty Liver Disease. Clin. Liver Dis. 2016, 20, 325-338. [CrossRef]

3. Betancourt-Garcia, M.M.; Arguelles, A.; Montes, J.; Hernandez, A.; Singh, M.; Forse, R.A. Pediatric Nonalcoholic Fatty Liver Disease: The Rise of a Lethal Disease Among Mexican American Hispanic Children. Obes. Surg. 2017, 27, 236-244. [CrossRef]

4. Crespo, M.; Lappe, S.; Feldstein, A.E.; Alkhouri, N. Similarities and differences between pediatric and adult nonalcoholic fatty liver disease. Metab. Clin. Exp. 2016, 65, 1161-1171. [CrossRef]

5. Castillo-Leon, E.; Cioffi, C.E.; Vos, M.B. Perspectives on youth-onset nonalcoholic fatty liver disease. Endocrinol. Diabetes Metab. 2020, 3, e00184. [CrossRef]

6. Milman, N.; Paszkowski, T.; Cetin, I.; Castelo-Branco, C. Supplementation during pregnancy: Beliefs and science. Gynecol. Endocrinol. 2016, 32, 509-516. [CrossRef]

7. Dai, X.; Guo, Z.; Chen, D.; Li, L.; Song, X.; Liu, T.; Jin, G.; Li, Y.; Liu, Y.; Ajiguli, A.; et al. Maternal sucralose intake alters gut microbiota of offspring and exacerbates hepatic steatosis in adulthood. Gut Microbes 2020, 11, 1043-1063. [CrossRef] [PubMed]

8. Thorn, S.R.; Baquero, K.C.; Newsom, S.A.; El Kasmi, K.C.; Bergman, B.C.; Shulman, G.I.; Grove, K.L.; Friedman, J.E. Early life exposure to maternal insulin resistance has pe rsistent effects on hepatic NAFLD in juvenile nonhuman primates. Diabetes 2014, 63, 2702-2713. [CrossRef]

9. Liem, D.G. Infants' and Children's Salt Taste Perception and Liking: A Review. Nutrients 2017, 9, 11. [CrossRef]

10. Mozaffarian, D.; Fahimi, S.; Singh, G.M.; Micha, R.; Khatibzadeh, S.; Engell, R.E.; Lim, S.; Danaei, G.; Ezzati, M.; Powles, J. Global sodium consumption and death from cardiovascular causes. N. Engl. J. Med. 2014, 371, 624-634. [CrossRef] [PubMed]

11. Reynolds, C.M.; Vickers, M.H.; Harrison, C.J.; Segovia, S.A.; Gray, C. High fat and/or high salt intake during pregnancy alters maternal meta-inflammation and offspring growth and metabolic profiles. Physiol. Rep. 2014, 2, e12110. [CrossRef]

12. Lee, E.; Kim, N.; Kang, J.; Yoon, S.; Lee, H.A.; Jung, H.; Kim, S.H.; Kim, I. Activated pathogenic Th17 lymphocytes induce hypertension following high-fructose intake in Dahl salt-sensitive but not Dahl salt-resistant rats. Disease Models Mech. 2020, 13. [CrossRef]

13. Marion, A.; Baker, A.; Dhawan, A. Fatty liver disease in children. Arch Dis. Child. 2004, 89, 648-652. [CrossRef] [PubMed]

14. Zhao, Y.C.; Zhao, G.J.; Chen, Z.; She, Z.G.; Cai, J.; Li, H. Nonalcoholic Fatty Liver Disease: An Emerging Driver of Hypertension. Hypertension 2020, 75, 275-284. [CrossRef] [PubMed]

15. Choi, Y.; Lee, J.E.; Chang, Y.; Kim, M.K.; Sung, E.; Shin, H.; Ryu, S. Dietary sodium and potassium intake in relation to non-alcoholic fatty liver disease. Br. J. Nutr. 2016, 116, 1447-1456. [CrossRef] [PubMed]

16. Zhang, H.Y.; Tian, J.X.; Lian, F.M.; Li, M.; Liu, W.K.; Zhen, Z.; Liao, J.Q.; Tong, X.L. Therapeutic mechanisms of traditional Chinese medicine to improve metabolic diseases via the gut microbiota. Biomed. Pharmacother. Biomed. Pharmacother. 2020, $133,110857$. [CrossRef]

17. Schwimmer, J.B.; Johnson, J.S.; Angeles, J.E.; Behling, C.; Belt, P.H.; Borecki, I.; Bross, C.; Durelle, J.; Goyal, N.P.; Hamilton, G.; et al. Microbiome Signatures Associated With Steatohepatitis and Moderate to Severe Fibrosis in Children With Nonalcoholic Fatty Liver Disease. Gastroenterology 2019, 157, 1109-1122. [CrossRef]

18. García-Mantrana, I.; Selma-Royo, M.; González, S.; Parra-Llorca, A.; Martínez-Costa, C.; Collado, M.C. Distinct maternal microbiota clusters are associated with diet during pregnancy: Impact on neonatal microbiota and infant growth during the first 18 months of life. Gut Microbes 2020, 11, 962-978. [CrossRef]

19. Wang, S.; Egan, M.; Ryan, C.A.; Boyaval, P.; Dempsey, E.M.; Ross, R.P.; Stanton, C. A good start in life is important-perinatal factors dictate early microbiota development and longer term maturation. FEMS Microbiol. Rev. 2020, 44, 763-781. [CrossRef]

20. Mohammad, M.A.; Sunehag, A.L.; Haymond, M.W. Effect of dietary macronutrient composition under moderate hypocaloric intake on maternal adaptation during lactation. Am. J. Clin. Nutr. 2009, 89, 1821-1827. [CrossRef]

21. Wang, C.; Huang, Z.; Yu, K.; Ding, R.; Ye, K.; Dai, C.; Xu, X.; Zhou, G.; Li, C. High-Salt Diet Has a Certain Impact on Protein Digestion and Gut Microbiota: A Sequencing and Proteome Combined Study. Front. Microbiol. 2017, 8, 1838. [CrossRef] [PubMed]

22. Soderborg, T.K.; Clark, S.E.; Mulligan, C.E.; Janssen, R.C.; Babcock, L.; Ir, D.; Young, B.; Krebs, N.; Lemas, D.J.; Johnson, L.K.J.N.c. The gut microbiota in infants of obese mothers increases inflammation and susceptibility to NAFLD. Nat. Commun. 2018, 9, 1-12. [CrossRef] [PubMed] 
23. Wahlström, A.; Sayin, S.I.; Marschall, H.U.; Bäckhed, F. Intestinal Crosstalk between Bile Acids and Microbiota and Its Impact on Host Metabolism. Cell Metab. 2016, 24, 41-50. [CrossRef]

24. Park, M.Y.; Kim, S.J.; Ko, E.K.; Ahn, S.H.; Seo, H.; Sung, M.K. Gut microbiota-associated bile acid deconjugation accelerates hepatic steatosis in ob/ob mice. J. Appl. Microbiol. 2016, 121, 800-810. [CrossRef]

25. Wang, Z.; Litterio, M.C.; Müller, M.; Vauzour, D.; Oteiza, P.I. (-)-Epicatechin and NADPH oxidase inhibitors prevent bile acid-induced Caco-2 monolayer permeabilization through ERK1/2 modulation. Redox Biol. 2020, 28, 101360. [CrossRef]

26. Jiao, N.; Baker, S.S.; Chapa-Rodriguez, A.; Liu, W.; Nugent, C.A.; Tsompana, M.; Mastrandrea, L.; Buck, M.J.; Baker, R.D.; Genco, R.J.J.G. Suppressed hepatic bile acid signalling despite elevated production of primary and secondary bile acids in NAFLD. Gut 2018, 67, 1881-1891. [CrossRef]

27. Mouries, J.; Brescia, P.; Silvestri, A.; Spadoni, I.; Sorribas, M.; Wiest, R.; Mileti, E.; Galbiati, M.; Invernizzi, P.; Adorini, L.; et al. Microbiota-driven gut vascular barrier disruption is a prerequisite for non-alcoholic steatohepatitis development. J. Hepatol. 2019, 71, 1216-1228. [CrossRef]

28. Nier, A.; Engstler, A.J.; Maier, I.B.; Bergheim, I. Markers of intestinal permeability are already altered in early stages of nonalcoholic fatty liver disease: Studies in children. PLoS ONE 2017, 12, e0183282. [CrossRef]

29. Carr, R.M.; Reid, A.E. FXR agonists as therapeutic agents for non-alcoholic fatty liver disease. Curr. Atheroscler. Rep. 2015, 17, 500. [CrossRef]

30. Alkhouri, N.; De Vito, R.; Alisi, A.; Yerian, L.; Lopez, R.; Feldstein, A.E.; Nobili, V. Development and validation of a new histological score for pediatric non-alcoholic fatty liver disease. J. Hepatol. 2012, 57, 1312-1318. [CrossRef] [PubMed]

31. Navas-Molina, J.A.; Peralta-Sánchez, J.M.; González, A.; McMurdie, P.J.; Vázquez-Baeza, Y.; Xu, Z.; Ursell, L.K.; Lauber, C.; Zhou, H.; Song, S.J.; et al. Advancing our understanding of the human microbiome using QIIME. Methods Enzymol. 2013, 531, $371-444$. [CrossRef]

32. Bustin, S.A.; Benes, V.; Garson, J.A.; Hellemans, J.; Huggett, J.; Kubista, M.; Mueller, R.; Nolan, T.; Pfaffl, M.W.; Shipley, G.L.; et al. The MIQE guidelines: Minimum information for publication of quantitative real-time PCR experiments. Clin. Chem. 2009, 55, 611-622. [CrossRef] [PubMed]

33. Chapin, R.E.; Robbins, W.A.; Schieve, L.A.; Sweeney, A.M.; Tabacova, S.A.; Tomashek, K.M.J.E.H.P. Off to a good start: The influence of pre-and periconceptional exposures, parental fertility, and nutrition on children's health. Environ. Health Perspect. 2004, 112, 69-78. [CrossRef]

34. Hao, H.; Cao, L.; Jiang, C.; Che, Y.; Zhang, S.; Takahashi, S.; Wang, G.; Gonzalez, F.J. Farnesoid X Receptor Regulation of the NLRP3 Inflammasome Underlies Cholestasis-Associated Sepsis. Cell Metab. 2017, 25, 856-867.e855. [CrossRef] [PubMed]

35. Huang, X.F.; Zhao, W.Y.; Huang, W.D. FXR and liver carcinogenesis. Acta Pharmacol. Sin. 2015, 36, 37-43. [CrossRef]

36. Sandboge, S.; Perälä, M.-M.; Salonen, M.K.; Blomstedt, P.A.; Osmond, C.; Kajantie, E.; Barker, D.J.; Eriksson, J.G.J.A.o.m. Early growth and non-alcoholic fatty liver disease in adulthood-The NAFLD liver fat score and equation applied on the Helsinki Birth Cohort Study. Ann. Med. 2013, 45, 430-437. [CrossRef] [PubMed]

37. Newton, K.P.; Feldman, H.S.; Chambers, C.D.; Wilson, L.; Behling, C.; Clark, J.M.; Molleston, J.P.; Chalasani, N.; Sanyal, A.J.; Fishbein, M.H.; et al. Low and High Birth Weights Are Risk Factors for Nonalcoholic Fatty Liver Disease in Children. J. Pediatrics 2017, 187, 141-146.e141. [CrossRef]

38. Dowla, S.; Aslibekyan, S.; Goss, A.; Fontaine, K.; Ashraf, A.P. Dyslipidemia is associated with pediatric nonalcoholic fatty liver disease. J. Clin. Lipidol. 2018, 12, 981-987. [CrossRef]

39. Yang, Z.X.; Shen, W.; Sun, H. Effects of nuclear receptor FXR on the regulation of liver lipid metabolism in patients with non-alcoholic fatty liver disease. Hepatol. Int. 2010, 4, 741-748. [CrossRef]

40. Watanabe, M.; Houten, S.M.; Wang, L.; Moschetta, A.; Mangelsdorf, D.J.; Heyman, R.A.; Moore, D.D.; Auwerx, J. Bile acids lower triglyceride levels via a pathway involving FXR, SHP, and SREBP-1c. J. Clin. Investig. 2004, 113, 1408-1418. [CrossRef] [PubMed]

41. Shimpi, P.C.; More, V.R.; Paranjpe, M.; Donepudi, A.C.; Goodrich, J.M.; Dolinoy, D.C.; Rubin, B.; Slitt, A.L. Hepatic Lipid Accumulation and Nrf2 Expression following Perinatal and Peripubertal Exposure to Bisphenol A in a Mouse Model of Nonalcoholic Liver Disease. Environ. Health Perspect. 2017, 125, 087005. [CrossRef] [PubMed]

42. Chen, X.; Zhang, C.; Zhao, M.; Shi, C.E.; Zhu, R.M.; Wang, H.; Zhao, H.; Wei, W.; Li, J.B.; Xu, D.X. Melatonin alleviates lipopolysaccharide-induced hepatic SREBP-1c activation and lipid accumulation in mice. J. Pineal Res. 2011, 51, 416-425. [CrossRef]

43. Li, Y.C.; Qiao, J.Y.; Wang, B.Y.; Bai, M.; Shen, J.D.; Cheng, Y.X. Paeoniflorin Ameliorates Fructose-Induced Insulin Resistance and Hepatic Steatosis by Activating LKB1/AMPK and AKT Pathways. Nutrients 2018, 10, 24. [CrossRef]

44. Madduma Hewage, S.; Prashar, S.; Siow, Y.L. Lingonberry Improves Non-Alcoholic Fatty Liver Disease by Reducing Hepatic Lipid Accumulation, Oxidative Stress and Inflammatory Response. Antioxidants 2021, 10, 565. [CrossRef] [PubMed]

45. Hatton, G.; Alterio, T.; Nobili, V.; Mann, J.P. Unmet needs in pediatric NAFLD research: What do we need to prioritize for the future? Expert Rev. Gastroenterol. Hepatol. 2018, 12, 961-967. [CrossRef] [PubMed]

46. Horton, J.D.; Goldstein, J.L.; Brown, M.S. SREBPs: Activators of the complete program of cholesterol and fatty acid synthesis in the liver. J. Clin. Investig. 2002, 109, 1125-1131. [CrossRef] [PubMed]

47. De Minicis, S.; Rychlicki, C.; Agostinelli, L.; Saccomanno, S.; Candelaresi, C.; Trozzi, L.; Mingarelli, E.; Facinelli, B.; Magi, G.; Palmieri, C.; et al. Dysbiosis contributes to fibrogenesis in the course of chronic liver injury in mice. Hepatology 2014, 59, 1738-1749. [CrossRef] 
48. Cui, Y.; Wang, Q.; Chang, R.; Zhou, X.; Xu, C. Intestinal Barrier Function-Non-alcoholic Fatty Liver Disease Interactions and Possible Role of Gut Microbiota. J. Agric. Food Chem. 2019, 67, 2754-2762. [CrossRef]

49. de Sant'Ana, L.P.; Ribeiro, D.J.S.; Martins, A.M.A.; Dos Santos, F.N.; Corrêa, R.; Almeida, R.D.N.; Eberlin, M.N.; Maurice, C.F.; Magalhães, K.G. Absence of the Caspases 1/11 Modulates Liver Global Lipid Profile and Gut Microbiota in High-Fat-Diet-Induced Obese Mice. Front. Immunol. 2019, 10, 2926. [CrossRef]

50. Shin, N.R.; Whon, T.W.; Bae, J.W. Proteobacteria: Microbial signature of dysbiosis in gut microbiota. Trends Biotechnol. 2015, 33, 496-503. [CrossRef]

51. Culligan, E.P.; Marchesi, J.R.; Hill, C.; Sleator, R.D. Combined metagenomic and phenomic approaches identify a novel salt tolerance gene from the human gut microbiome. Front. Microbiol. 2014, 5, 189. [CrossRef]

52. Boursier, J.; Mueller, O.; Barret, M.; Machado, M.; Fizanne, L.; Araujo-Perez, F.; Guy, C.D.; Seed, P.C.; Rawls, J.F.; David, L.A.; et al. The severity of nonalcoholic fatty liver disease is associated with gut dysbiosis and shift in the metabolic function of the gut microbiota. Hepatology 2016, 63, 764-775. [CrossRef]

53. Do, M.H.; Lee, H.B.; Lee, E.; Park, H.Y. The Effects of Gelatinized Wheat Starch and High Salt Diet on Gut Microbiota and Metabolic Disorder. Nutrients 2020, 12, 301. [CrossRef]

54. Li, W.; Zhang, K.; Yang, H. Pectin Alleviates High Fat (Lard) Diet-Induced Nonalcoholic Fatty Liver Disease in Mice: Possible Role of Short-Chain Fatty Acids and Gut Microbiota Regulated by Pectin. J. Agric. Food Chem. 2018, 66, 8015-8025. [CrossRef]

55. Zhang, X.; Dong, Y.; Sun, G.; Hasan, A.A.; Tian, M.; Zeng, S.; Li, J.; Zeng, S.; Ma, J.; Li, J.; et al. Paternal Programming of Liver Function and Lipid Profile Induced by a Paternal Pre-Conceptional Unhealthy Diet: Potential Association with Altered Gut Microbiome Composition. Kidney Blood Press. Res. 2019, 44, 133-148. [CrossRef] [PubMed]

56. Do, M.H.; Lee, H.B.; Oh, M.J.; Jhun, H.; Ha, S.K.; Park, H.Y. Consumption of salt leads to ameliorate symptoms of metabolic disorder and change of gut microbiota. Eur. J. Nutr. 2020, 59, 3779-3790. [CrossRef] [PubMed]

57. Chen, J.; Thomsen, M.; Vitetta, L. Interaction of gut microbiota with dysregulation of bile acids in the pathogenesis of nonalcoholic fatty liver disease and potential therapeutic implications of probiotics. J. Cell. Biochem. 2019, 120, 2713-2720. [CrossRef]

58. Zeng, H.; Larson, K.J.; Cheng, W.H.; Bukowski, M.R.; Safratowich, B.D.; Liu, Z.; Hakkak, R. Advanced liver steatosis accompanies an increase in hepatic inflammation, colonic, secondary bile acids and Lactobacillaceae/Lachnospiraceae bacteria in C57BL/6 mice fed a high-fat diet. J. Nutr. Biochem. 2020, 78, 108336. [CrossRef] [PubMed]

59. Stenman, L.K.; Holma, R.; Eggert, A.; Korpela, R. A novel mechanism for gut barrier dysfunction by dietary fat: Epithelial disruption by hydrophobic bile acids. Am. J. Physiol. Gastrointest. Liver Physiol. 2013, 304, G227-C234. [CrossRef] [PubMed]

60. Li, Q.; Li, M.; Li, F.; Zhou, W.; Dang, Y.; Zhang, L.; Ji, G. Qiang-Gan formula extract improves non-alcoholic steatohepatitis via regulating bile acid metabolism and gut microbiota in mice. J. Ethnopharmacol. 2020, 258, 112896. [CrossRef] [PubMed]

61. Ma, J.; Hong, Y.; Zheng, N.; Xie, G.; Lyu, Y.; Gu, Y.; Xi, C.; Chen, L.; Wu, G.; Li, Y.; et al. Gut microbiota remodeling reverses aging-associated inflammation and dysregulation of systemic bile acid homeostasis in mice sex-specifically. Gut Microbes 2020, 11, 1450-1474. [CrossRef]

62. Hu, J.; Luo, H.; Wang, J.; Tang, W.; Lu, J.; Wu, S.; Xiong, Z.; Yang, G.; Chen, Z.; Lan, T.; et al. Enteric dysbiosis-linked gut barrier disruption triggers early renal injury induced by chronic high salt feeding in mice. Exp. Mol. Med. 2017, 49, e370. [CrossRef] [PubMed]

63. Zhang, Z.; Zhao, J.; Tian, C.; Chen, X.; Li, H.; Wei, X.; Lin, W.; Zheng, N.; Jiang, A.; Feng, R.; et al. Targeting the Gut Microbiota to Investigate the Mechanism of Lactulose in Negating the Effects of a High-Salt Diet on Hypertension. Mol. Nutr. Food Res. 2019, 63, e1800941. [CrossRef] [PubMed]

64. Jena, P.K.; Sheng, L.; Liu, H.X.; Kalanetra, K.M.; Mirsoian, A.; Murphy, W.J.; French, S.W.; Krishnan, V.V.; Mills, D.A.; Wan, Y.Y. Western Diet-Induced Dysbiosis in Farnesoid X Receptor Knockout Mice Causes Persistent Hepatic Inflammation after Antibiotic Treatment. Am. J. Pathol. 2017, 187, 1800-1813. [CrossRef] [PubMed]

65. Sayin, S.I.; Wahlström, A.; Felin, J.; Jäntti, S.; Marschall, H.U.; Bamberg, K.; Angelin, B.; Hyötyläinen, T.; Orešič, M.; Bäckhed, F. Gut microbiota regulates bile acid metabolism by reducing the levels of tauro-beta-muricholic acid, a naturally occurring FXR antagonist. Cell Metab. 2013, 17, 225-235. [CrossRef]

66. Liu, H.; Pathak, P.; Boehme, S.; Chiang, J.Y. Cholesterol $7 \alpha$-hydroxylase protects the liver from inflammation and fibrosis by maintaining cholesterol homeostasis. J. Lipid Res. 2016, 57, 1831-1844. [CrossRef] [PubMed] 